\title{
MEDIDAS QUANTITATIVAS CLÍNICAS E ELETRENCEFALOGRÁFICAS DE EPILEPTOGÊNESE NA EPILEPSIA DO LOBO TEMPORAL
}

\author{
P.R.M. DE BITTENCOURT ${ }^{*}$ A.C. GABARDO**, T. MARCOURAKIS ${ }^{\star \star *}$
}

\begin{abstract}
RESUMO - Neste estudo controlado, prospectivo e parcialmente cego foram avaliados dois grupos de 10 portadores de epilepsia do lobo temporal, respectivamente de bom e mau prognóstico. Foram definidas medidas de epileptogênese baseadas nas frequências de crises e na quantificação de atividade epileptogênica nas derivações eletrencefalográficas de lobo temporal. Os grupos de estudo foram semelhantes a no mínimo um dos grupos de controle em idade, sexo, nível educacional e social, regime terapêutico, idade de início e tempo de evolução da epilepsia. Houve uma diferença global do grupo de epilepsia do lobo temporal com mau prognóstico das outras populações, atingindo significação estatística em epileptogênese clínica, e uma tendência dos dados sugerindo maior epileptogênese eletrencefalográfica na mesma população. Os resultados indicam a utilidade experimental de algumas das medidas introduzidas, mas também seus problemas. Revisão da literatura pertinente é realizada.
\end{abstract}

PALAVRAS-CHAVE: epilepsia do lobo temporal, epileptogênese, EEG.

\section{Quantitative clinical and EEG measures of epileptogenesis in temporal lobe epilepsy}

SUMMARY - In this controlled, prospective and partially blind study two groups of patients with temporal lobe epilepsy were evaluated, respectively with good and bad prognosis. Measurements of epileptogenesis were based on frequency of seizures, and on epiletogenic eletroencephalographic abnormalities obtained from scalp electrodes over the temporal lobes. The results were analised by non-parametric analysis of variance, comparison of groups and analysis of correlation. The results indicated the temporal lobe groups were similar to at least one of the control groups in age, sex, educational and social level, therapeutic regime, age at onset and length of history of epilepsy. The quantitative measurements showed a global difference between the group of temporal lobe with bad and good prognosis, reaching statistical significance in clinical epileptogenesis, and a trend towards greater epileptogenesis on the eletroencephalogram, in the same group of patients. The results indicate the experimental usefulness of some of the original measurements used in the study, but also their problems. A review of the literature is carried out.

KEY WORDS: temporal lobe epilepsy, epileptogenesis, EEG.

As crises da epilepsia do lobo temporal são, por definição, parciais simples e/ou complexas com ou sem generalização secundária ${ }^{13,14}$. As crises têm algumas características gerais como a ocorrência em "cachos" e o início com sintomas autonômicos, psíquicos e/ou sensitivos, na aura. As crises parciais complexas mais típicas começam com uma "parada motora", seguida de automatismos.

Unidades de Neurologia Clínica $\left({ }^{*}\right)$ e Tomografia Computadorizada $\left({ }^{* *}\right)$, do Hospital Nossa Senhora das Graças, Curitiba; Centro de Investigações em Neurologia $(* * *)$ da Faculdade de Medicina da Universidade de São Paulo. Estudo apresentado em parte como Tese (PRMB) aprovada em Concurso para Professor Titular de Doenças do Sistema Nervoso na Universidade Federal do Paraná (UFPR) em 1991. Aceite: 12-dezembro-1994.

Dr. Paulo Rogério Mudrovitsch de Bittencourt - Unidade de Neurologia Clínica S/C Ltda - Rua Padre Anchieta 155 80410-030 Curitiba PR - Brasil. 
As crises do lobo temporal se subdividem em amígdalo-hipocampais (mesiais) e neocorticais ou laterais. As primeiras têm sintomas como desconforto epigástrico, borborigmos, eructações, palidez ou vermelhidão do rosto, parada respiratória, dilatação pupilar, medo, pânico e alucinações olfativogustatórias. As crises temporais laterais envolvem audição, visão e linguagem no caso do hemisfério dominante $\mathrm{e}^{13,14}$ e podem evoluir para crises parciais complexas integrais quando estruturas extratemporais são envolvidas. As crises amígdalo-hipocampais evoluem com maior frequência para crises tônico-clônicas secundárias ${ }^{13,14}$.

\section{MEDIDAS DE INTENSIDADE DA EPILEPSIA}

Uma boa fonte de observações sobre medidas de intensidade da epilepsia são os estudos de drogas introduzidas na prática médica nos últimos 10 anos, muitas ainda em experimentação clínica ${ }^{29}$. Normas estritas para estes estudos foram publicadas pela Liga Internacional Contra a Epilepsia ${ }^{12}$. Uma boa revisão das técnicas de monitorização da gravidade e/ou intensidade das epilepsias foi feita por Porter e White ${ }^{32}$.

As variáveis estudadas são sempre crises epilépticas, definidas de acordo com a classificação internacional de $1981^{12,15}$. As crises mais facilmente analisáveis, principalmente por ter reflexo eletrencefalográfico reproduzível e específico, são as ausências das epilepsias generalizadas idiopáticas. Outro tipo de crise frequentemente utilizada é a tônico-clônica. Sua fenomenologia óbvia a torna medida extremamente confiável do controle da epilepsia. As crises parciais são a outra variável utilizada. Suas vantagens são a maior frequência no indivíduo e na população. Além disso, sua ocorrência, embora clara, gera menos ansiedade que crises tônico-clônicas, eliminando fator emocional importante dos estudos longitudinais, com duração de vários meses. As desvantagens das crises parciais são sua heterogeneidade, principalmente em grupos grandes de pacientes e, principalmente, quando crises parciais simples e complexas são analisadas conjuntamente ${ }^{32}$. Em nosso meio, está bem demonstrado que crises parciais são diagnosticáveis tanto em adultos ${ }^{4}$ quanto em crianças atendidas em hospitais-escola ${ }^{38}$.

Estudos recentes realizados para avaliar a eficácia e tolerabilidade da vigabatrina ${ }^{11,31}$, lamotrigina ${ }^{3,24}$ e oxcarbazepina ${ }^{16}$ colaboraram muito na verificação de métodos de quantificação de crises parciais simples e complexas, utilizando a classificação internacional de crises ${ }^{15}$. Fica claro que a quantificação dessas crises reflete o efeito da medicação no processo epileptogênico ${ }^{8,25} \mathrm{e}$ que existe suficiente especificidade e acordo entre investigadores em diferentes centros utilizando o mesmo método de quantificação ${ }^{31}$ (Bittencourt et al, enviado para publicação). Assim, parece justificada a utilização da simples contagem de crises epilépticas parciais simples e complexas, com ou sem generalização secundária, como medida de intensidade da epilepsia do lobo temporal ${ }^{12,32}$.

Por outro lado, está bem estabelecido já há vários anos que o EEG não é bom método de monitorização da intensidade do processo epileptogênico ${ }^{30,32}$. Tentativas mais específicas de quantificar objetivamente atividade epileptiforme de lobo temporal como medida do efeito antiepiléptico de certas drogas ${ }^{2}$ tiveram sucesso parcial. Embora haja grande variabilidade intra e inter-individual na frequência e magnitude de pontas epileptiformes interictais, uma avaliação subjetiva pode dar idéia quantitativa da intensidade da epileptogênese do lobo temporal ${ }^{36}$. Tentativas de quantificar, mesmo que subjetivamente, outras anormalidades eletrencefalográficas associadas à epilepsia do lobo temporal têm grande tendência de levar à frustação. Primeiramente, porque existem certos ritmos eletrencefalográficos aparentemente anormais, mas que claramente não têm relação com epileptogênese ${ }^{40}$. Em segundo lugar, porque mesmo no caso de lentificação localizada do EEG, um padrão reconhecidamente associado à epilepsia do lobo temporal ${ }^{15,36}$, pode haver grande discordância entre eletrencefalografistas sobre sua quantificação e significado ${ }^{33}$. Existe certo consenso ${ }^{36}$ sobre a relação entre atividade epileptiforme no EEG e prognóstico nas epilepsias localizadas sintomáticas: o prognóstico parece ser pior na população com EEG anormal quando comparada com EEG epileptiforme. 
Nas epilepsias generalizadas idiopáticas, em que o padrão epileptiforme de complexos pontaonda e complexos poliponta-onda é extremamente óbvio, confiável e reproduzível, a quantificação pode ser feita facilmente pela contagem manual ou automática, por computador, do número de complexos ou do tempo passado pelo paciente em complexos ponta-onda ${ }^{37}$. Existe correlação entre complexos ponta-onda e as ausências simples das epilepsias generalizadas idiopáticas, mas não com respeito às ausências complexas, crises mioclônicas ou outras crises que ocorrem com epilepsias generalizadas idiopáticas, criptogenéticas ou sintomáticas ${ }^{12,32}$.

\section{MÉTODOS}

Este estudo fez parte de um experimento prospectivo e controlado, "seccional-transverso" (do inglês, "a cross-sectional study"), de 2 grupos de 10 portadores de epilepsia do lobo temporal, diferenciados pela gravidade maior ou menor da epilepsia. Todos os pacientes foram atendidos entre 1982 e 1990 na Unidade de Neurologia Clínica S/C Ltda. Todos foram reclassificados de acordo com suas crises ${ }^{15}$ e síndromes ${ }^{13,14}$ pois, em muitos casos, a especificidade anatômica da classificação das epilepsias do lobo temporal não havia sido empregada no atendimento clínico de maneira completa.

Os critérios de inclusão para os portadores de epilepsia do lobo temporal foram: nível sócio-econômico médio ou maior; nível cultural que incluísse alfabetização completa; diagnóstico clínico de epilepsia com localização sintomática ou criptogênica ${ }^{13,14} \mathrm{com}$ crises parciais simples ou complexas do lobo temporal ${ }^{13-15}$ documentadas clinicamente de maneira satisfatória durante período de seguimento de pelo menos 6 meses; pelo menos 2 EEGs considerados satisfatórios realizados nos últimos 10 anos demosntrando padrão epileptiforme inconfundível de epilepsia do lobo temporal ${ }^{15,36}$. Foram considerados critérios de exclusão: sinais clínicos ou em estudos de imagem indicativos de lesão estrutural cerebral progressiva ou de lesão cerebral estável significante neurologicamente, istoé, suficiente para produzir sequelas neurológicas; distúrbio neuropsiquiátrico clinicamente relevante, com retardo mental claro na avaliação neurológica, com QI presumível abaixo de 60; qualquer dúvida sobre a etiologia epiléptica das crises apresentadas pelos pacientes.

Foram estudados 2 grupos de portadores de epilepsia do lobo temporal: um deles chamado de epilepsia do lobo temporal de "bom prognóstico", com sua epilepsia em remissão; e outro de "mau prognóstico", com a epilepsia em atividade. No primeiro grupo foram incluídos pacientes com menos de duas crises parciais simples ou complexas ao mês e tratamento com uma só droga antiepiléptica (valproato de sódio, carbamazepina ou fenitoína). No segundo grupo foram incluídos pacientes com mais de 4 crises parciais simples ou complexas ao mês, inclusive no último mês antes do estudo, sob tratamento com mais de uma droga antiepiléptica. A presença de outros tipos de crise epiléptica, como por exemplo crises tônico-clônicas, foi considerada indicação de epilepsia "em atividade".

MEDIDAS DE INTENSIDADE DA EPILEPSIA. A primeira medida, chamada intensidade cumulativa, foi graduada entre 1 e 4 de acordo com o número global de crises que a pessoa teria tido em toda sua vida, desde o início da doença. Assim, o grau 1 foi menos de 20 crises parciais; o grau 2, entre 20 e 100 crises parciais, além de menos de 10 crises tônico-clônicas secundariamente generalizadas; o grau 3, entre 100 e 500 crises parciais e menos de 50 tônico-clônicas; o grau 4, mais de 500 crises parciais e mais de 50 tônico-clônicas. Uma segunda medida de intensidade passada da epilepsia foi a frequência média mensal global, isto é, de todos os tipos de crises, calculada para toda a vida do paciente até 6 meses antes do estudo. A medida de intensidade atual da epilepsia foi dada pela frequência média global das crises nos últimos 6 meses. As mesmas medidas foram também aplicadas ao grupo de controles com epilepsia generalizada idiopática. A medida de frequência atual mensal de crises foi também quantificada entre 1 e 4 , respectivamente quando fosse entre 0 e 3,4 e 10, 11 e 50 , ou mais de 50 crises ao mês.

EPILEPTOGÊNESE: TÉCNICA ELETRENCEFALOGRÁFICA. Os traçados de EEG foram obtidos em condições usuais. Os traçados foram sempre feitos pela manhã, após uma noite de privação máxima de sono, ou seja, após os indivíduos terem sidos instruídos para não dormir nada ou o mínimo possível pela utilização de meios de lazer. Em todos os casos foram obtidos pelo menos 20 minutos de traçado em vigília e 20 minutos em sono espontâneo, conforme normas tradicionais da Federação Internacional das Sociedades de Eletrencefalografia e Neurofisiologia Clínica. O aparelho utilizado foi um eletrencefalógrafo GRASS (Quincy, Massachusetts) modelo 6, de 8 canais. Os traçados foram obtidos através do Sistema Internacional 10-20 de colocação de eletrodos. Especial ênfase foi dada às derivações de lobo temporal, através da utilização da montagem IX 
Tabela 1. Idade de início e tempo de evolução das epilepsias.

\begin{tabular}{|c|c|c|c|c|c|c|c|c|c|c|}
\hline & \multicolumn{5}{|c|}{ Idade de início } & \multicolumn{5}{|c|}{ Tempo de evolução } \\
\hline & MIN & MAX & MED & MNA & DP & MIN & MAX & MED & MNA & DP \\
\hline B & 7 & 52 & 19 & 11,5 & 16 & 0,7 & 40 & 20 & 17,5 & 13 \\
\hline $\mathbf{M}$ & 2 & 17 & 11 & 12,5 & 5 & 8 & 42 & 24 & 22,5 & 9 \\
\hline
\end{tabular}

B, lobo temporal com bom prognóstico; M, lobo temporal com mau prognóstico; valores expressos em anos.

durante todo o traçado. Estas derivações são respectivamente : Fz-F8; F8-F7; F7-Fz; Cz-T4; T4-T3; T3-Cz; Fz$\mathrm{Cz}$; 02-01. O índice eletrencefalográfico de epileptogênese foi graduado em: 1, traçado normal; 2 , ondas agudas isoladas e raras, ou seja, um foco epileptiforme de características discretas; 3 , ondas agudas moderadamente frequentes, caracterizando um foco moderado; e 4, ondas agudas muito frequentes, ou um foco de características intensas.

\section{RESULTADOS}

Não houve diferença significativa de idade entre os grupos do lobo temporal $(p=0,60)$. As diferenças de frequência de sexo entre os grupos não foram significativas (teste exato de Fisher, $p>0,164$ ). Análise de variância pelo método de Kruskall-Wallis e comparação pelo método de Mann-Whitney mostraram não haver diferenças significativas entre as medidas do tempo de evolução do quadro clínico (Tabela 1).

Portadores de epilepsia de lobo temporal de bom prognóstico tinham síndrome amígdalo-hipocampal em 8 casos e temporal posterior lateral nos outros 2 casos. Destes, 1 paciente com síndrome amígdalo-hipocampal estava sendo tratado com fenitoína e todos os outros 9 com carbamazepina. No grupo de mau prognóstico, 9 tinham síndrome amígdalo-hipocampal e 1 tinha síndrome temporal posterior lateral. Neste grupo todos estavam tratados com mais de uma droga antiepiléptica. Uma das drogas era carbamazepina em 10 casos; a segunda droga era valproato de sódio em 5 casos; fenitoína e clonazepam em 2 casos cada; e vigabatrina em 1 caso. Duas pacientes estavam tomando uma terceira droga, vigabatrina e etosuximida, respectivamente. Não houve diferença entre as concentrações de carbamazepina entre os 2 grupos ( $p=0,205$, teste $t$ de Student para observações nãopareadas). Foi calculado ainda o número de drogas antiepilépticas por paciente nos grupos estudados. Este número vem a ser respectivamente 1 e 2,2 nos grupos de portadores de epilepsia do lobo temporal de bom e mau prognóstico.

\section{INTENSIDADE CLÍNICA DA EPILEPSIA}

As medidas utilizadas comprovaram ser úteis e práticas, com a possibilidade de consenso entre investigador e portador de epilepsia, com ou sem a cooperação de familiares.

A frequência atual de crises foi submetida a ANOVA pelo método de Kruskall-Wallis, que revelou não terem as populações a mesma mediana $(p<0,0001)$. O teste de Mann-Whitney revelou que os portadores de epilepsia do lobo temporal com mau prognóstico tinham mais crises que os do lobo temporal com bom prognóstico $(\mathrm{p}<0,0001)$. A frequência passada de crises foi submetida a análise de variância pelo método de KruskallWallis, que revelou terem as populações medianas diferentes $(p=0,0078)$. O teste de Mann-Whitney mostrou que a frequência foi maior no grupo de lobo temporal de mau prognóstico que no bom prognóstico ( $p=0,0021)$.

$O$ número cumulativo de crises foi submetido ao mesmo tipo de exploração estatística. A ANOVA por Kruskall-Wallis revelou diferença $(\mathrm{p}=0,0012)$, demonstrada pelo teste de Mann-Whitney entre os grupos do lobo temporal de bom e mau prognóstico $(\mathrm{p}=0,0017)$, sempre este último sendo maior.

Tabela 2. Frequência média mensal global de crises nos 6 meses antes do estudo, em portadores de epilepsia generalizada idiopática, do lobo temporal (Temp.) com bom e mau prognóstico.

\begin{tabular}{lccccc}
\hline & \multicolumn{5}{c}{ Intensidade atual da epilepsia } \\
\cline { 2 - 6 } & MIN & MAX & MED & MNA & DP \\
\hline \hline Temp. bom & 0 & 2 & 0,5 & 0,25 & 0,7 \\
Temp. mau & 4 & 31 & 10,4 & 8,75 & 8,1 \\
\hline
\end{tabular}


Tabela 3. Frequência média mensal global de crises nos pacientes com epilepsia generalizada idiopática de lobo temporal (Temp.) de bom e mau prognóstico, entre 18 e 12 meses antes do estudo.

\begin{tabular}{lccccc}
\hline & \multicolumn{5}{c}{ Intensidade passada da epilepsia } \\
\cline { 2 - 6 } & MIN & MAX & MED & MNA & DP \\
\hline \hline Temp. bom & 0,3 & 80 & 12 & 1,35 & 26 \\
Temp. mau & 3 & 100 & 13 & 10,25 & 11 \\
\hline
\end{tabular}

Tabela 4. Anormalidades eletrencefalográficas.

\begin{tabular}{lllc}
\hline Cod. & Nome & \multicolumn{1}{c}{ Anormalidade } & Intensidade \\
\hline B1 & SP & temporal direita & 3 \\
B2 & MVZB & temporal esquerda & 3 \\
B3 & MB & paroxismos lentos difusos & 1 \\
B4 & LGR & temporal direita & 2 \\
B5 & MBS & bitemporal independente + gen. secundária & 2 \\
B6 & RCK & temporal direita & 2 \\
B7 & ERC & bitemporal independente, E>D & 4 \\
B8 & RSC & temporal direita & 2 \\
B9 & MTMR & bitemporal independente & 2 \\
B10 & DNP & temporal esquerda & 2 \\
M1 & FCM & bitemporal independente & 3 \\
M2 & RMCK & temporal esquerda & 2 \\
M3 & JMJ & temporal direita & 2 \\
M4 & IOM & bitemporal independente & 3 \\
M5 & VNJ & bitemporal independente & 4 \\
M6 & EB & temporal direita & 2 \\
M7 & EC & bitemporal independente & 3 \\
M8 & CJG & temporal esquerda & 4 \\
M9 & MVSF & temporal esquerda & 2 \\
M10 & CTC & temporal esquerda & 3 \\
\hline
\end{tabular}

Código (B, bom prognóstico; M, mau prognóstico),iniciais (Nome), localização da anormalidade eletrencefalográfica e graduação subjetiva de intensidade (1, normal; 2, discreta; 3, moderada; 4, intensa; E, esquerda; D, direita; gen, generalização).

A quantificação de intensidade cumulativa da epilepsia mostrou que no grupo generalizado idiopático três indivíduos receberam grau 1, quatro grau 2,11 grau 3 e cinco grau 4. Esta mesma sequência mostrou, no grupo de portadores de epilepsia do lobo temporal com bom prognóstico, respectivamente nenhum, 3, 4 e 3 casos. No de mau prognóstico todos receberam grau 4. Análise estatística mostrou que a intensidade cumulativa foi maior no grupo de lobo temporal de mau prognóstico do que no de bom prognóstico ( $p=0,0003)$.

\section{INTENSIDADE ELETRENCEFALOGRÁFICA DA EPILEPSIA}

Os resultados qualitativos e quantitativos da análise eletrencefalográfica estão expressos na Tabela 4. Apenas um paciente, do grupo de bom apresentou prognóstico traçado sem características epileptogênicas nos lobos temporais. Um paciente, também do grupo de bom prognóstico, apresentou generalização secundária em ponta-onda, também chamada bissincronia bilateral secundária. No grupo de mau prognóstico todos os traçados foram epileptogênicos. Estas frequências não são diferentes entre si (teste exato de Fisher, p=0,5).

Quanto à lateralização das anormalidades, não ocorreram diferenças aparentes entre os 2 grupos. Análise estatística da graduação de intensidade eletrencefalográfica da epileptogênese mostrou medianas de 2 e 3 , respectivamente nos grupos de bom e mau prognóstico, que vem a ser uma diferença no limite da significação estatística $(p=0,0758)$. 


\section{COMENTÁRIOS}

A medida eletrencefalográfica de epileptogênese utilizada pode ser considerada bem sucedida, pelo menos relativamente. Em termos estatísticos, que usualmente não fazem parte desta disciplina das neurociências, foi quase possível separar os dois grupos de epilépticos do lobo temporal. Fica bastante provável que com número pouco maior de indivíduos, ou com grupos mais separados dentro do espectro de gravidade, teria sido significativa a diferença de intensidade eletrencefalográfica de epileptogênese entre os dois grupos de pacientes com epilepsia do lobo temporal.

As drogas que parecem ter efeito variável sobre o processo epileptogênico, entre elas benzodiazepínicos, barbitúricos, valproato de sódio e vigabatrina, têm em comum seu presumido mecanismo de ação através do complexo de receptores GABA, que incluem os receptores benzodiazepínico e barbitúrico e os canais de cloro ${ }^{19,21,22,26,28,42}$. No caso do receptor benzodiazepínico, a evolução no tempo do processo de tolerância já foi amplamente avaliada em animais ${ }^{27}$ e pode ser estudada em seres humanos através de $\mathrm{PET}^{39}$. A tolerância a barbitúricos também já foi amplamente avaliada em animais ${ }^{41}$.

Esta breve revisão e um amplo segmento da literatura indica que alguns dos nossos pacientes estavam, na época do estudo, sob tratamento com drogas de eventual potencial epileptogênico, quais sejam barbitúricos, benzodiazepínicos ou vigabatrina ${ }^{4-9,17,23,25,34}$. Havia duas pacientes em tratamento com vigabatrina e duas com clonazepam no grupo de mau prognóstico. No caso destas duas drogas, a epileptogênese eventualmente induzida pela medicação seria do tipo ponta-onda, ou seja, generalizada, facilmente perceptível no EEG, que não mostrou esta anormalidade durante o presente estudo. Isto se explica porque no caso da vigabatrina este efeito parece ser relacionado com dose $^{8}$ e os pacientes já haviam tido suas doses acertadas para que não ocorresse bissincronia bilateral secundária. No caso do clonazepam, as doses eram pequenas e, talvez por esta razão, não pareceu haver epileptogênese induzida. No caso da vigabatrina a manifestação clínica é discreta, na forma de mioclonias, e não seria detectada por qualquer das medidas de epileptogênese. Já no caso do clonazepam, poderiam ocorrer crises tônico-clônicas, mas esta não foi a impressão clínica no momento da inclusão desses pacientes no estudo.

Foi ressaltada pelos nossos resultados a dificuldade apresentada pelo EEG de rotina como método de lateralização da descarga epileptogênica. Grande parte dos pacientes apresentou, no EEG obtido para este experimento, lateralidade diferente de exames obtidos anteriormente. Este fato deve ser explicado por epileptogênese secundária que, em pacientes com epilepsia crônica, tende a causar manifestações bilaterais.

A maior dificuldade no diagnóstico de crises do lobo temporal é verificada com crises parciais simples ou complexas de lobo frontal. O conceito sindrômico de epilepsias do lobo frontal é recente e restrito a centros de cirurgia de epilepsia ou a centros com ampla tradição de monitorização intensiva de crises com circuito fechado de vídeo-EEG ${ }^{20}$. Até recentemente, embora existisse esta capacidade na América Latina ${ }^{35}$, não existia um grupo de regras ou critérios aceitos internacionalmente, que permitissem o diagnóstico de crises frontais durante a entrevista ou à beira do leito ${ }^{4,10}$. Uma revisão bibliográfica indica que crises frontais são caracterizadas: por durarem menos de 1 minuto; por ocorrerem em "cachos" de muitas crises ao dia e especialmente durante o sono; pela curiosidade da crise em si, com vocalizações obscenas, latinos, comportamentos sexuais, sintomas motores bizarros e frenéticos; por serem estereotipadas; e pelo rápido retorno da consciência (Delgado-Escueta et al ${ }^{18}$; Wolf, comunicação pessoal, 1990). Ainda em 1988, Bancaud, uma das maiores autoridades na história da localização cerebral da epileptogênese afirmou que "a principal questão a ser endereçada é se novos métodos de exploração do cérebro humano permitirão uma classificação anátomo-eletroclínica das crises do lobo frontal, com ou sem a ajuda do modelo utilizado para a classificação das crises de lobo temporal"'. A metodologia desenvolvida no presente estudo pode contribuir para o esclarecimento desta questão. 
Agradecimento-Os autores reconhecem o auxílio durante a execução deste estudo dos Drs. P.A. Kowacs, R.R. Seixas e A.F. Seixas, então médicos do Centro Diagnóstico de Curitiba.

\section{REFERÊNCIAS}

1. Bancaud J. Historical introduction to concepts and classification of frontal lobe seizures. Epilepsia 1988, 29: 204.

2. Binnie CD. The use of inter-ictal EEG in the study of antiepileptic drugs. In Buser PA, Cobb WA, Okuma T. Kyoto Symposia. Amsterdam: Elsevier 1982, p 504-512.

3. Binnie CD, Debets RMC, Engelsman M, Meijer JWA, Meinardi H, Overweg J, Peck AW, van Wieringen A, Yuen WC. Double-blind crossover trial of lamotrigine (Lamictal) as add-on therapy in intractable epilepsy. Epilepsy Res 1989, 4: 222-229.

4. Bittencourt PRM. Epilepsy in Latin America. In Laidlaw J, Richens A, Oxley J. A textbook of Epilepsy. Ed

3. Edinburgh: Churchill Livingstone, 1988, p 518-528.

5. Bittencourt PRM. May barbiturates be epiletogenic? In Lüders H. Second International Cleveland Clinic Epilepsy Symposium - Epilepsy Surgery: pathophysiology, clinical symptomatology, medical treatment, patient selection and surgical techniques, 1990, Cleveland: Cleveland Clinic Foundation, 1990, p 19.

6. Bittencourt PRM, Antoniuk SA, Costa JC, Fonseca LC, Gorz AM, Marcourakis T, Gonçalves e Silva GE. Carbamazepine and phenytoin in epilepsies refractory to barbiturates: efficacy, tolerability and mental function. Epilepsy Res 1993, 16: 147-155.

7. Bittencourt PRM, Dhillon S. Benzodiazepines: clinical aspects. In Richens A, Marks V. Therapeutic drug monitoring. Edinburgh: Churchill Livingstone, 1981, p 255-271.

8. Bittencourt PRM, Mazer S, Marcourakis T, Ferreira ZS, Reinhard AMG. Rational polytherapy for epilepsy. Epilepsia (na prensa).

9. Bittencourt PRM, Richens A. Case report: anticonvulsant-induced status epilepticus in Lennox-Gastaut syndrome. Epilepsia 1981, 22: 129-134.

10. Bittencourt PRM, Turner M. Latin American aspects. In Dam M, Gram L. Comprehensive epileptology. New York: Raven Press, 1990, p 807-820.

11. Browne TR, Mattson RH, Penry JK, Smith DB, Treiman DM, Wilder BJ, Ben-Menachem E, Napoliello MJ, Sherry KM, Szabo GK. Vigabatrin for refractory complex partial seizures: multicenter single-blind study with long-term follow-up. Neurology 1987, 37: 184-189.

12. Commission on Antiepileptic Drugs of the International League Against Epilepsy. Guidelines for clinical evaluation of antiepileptic drugs. Epilepsia 1989, 30: 400-408.

13. Commission on Classification and Terminology of the International League Against Epilepsy. Proposal for classification of epilepsies and epileptic syndromes. Epilepsia 1985, 26: 268-278.

14. Commission on Classification and Terminology of the International League Against Epilepsy. Proposal for revised classification of epilepsies syndromes. Epilepsia 1989, 30: 389-399.

15. Commission on Classification and Terminology of the International League Against Epilepsy. Proposal for revised clinical and eletroencephalographic classification of epileptic seizures. Epilepsia 1981, 22: 489-501.

16. Dam M, Ekberg R, Loyning Y, Waltimo O, Jakobsen K. A double-blind study comparing oxcarbazepine and carbamazepine in patients with newly diagnosed previously untreated epilepsy. Epilepsy Res 1989, 3: 70-76.

17. Dean C, Penry JK. Valproate monotherapy in 30 patients with partial seizures. Epilepsia 1987, 28: 605.

18. Delgado-Escueta AV, Chauvel P, Halgren E, Bancaud J. A NATO international advanced research workshop on frontal lobe seizures and epilepsies. Epilepsia 1988, 29: 204-221.

19. Dingledine R. NMDA receptors: what do they do? Trends Neurosci 1986, 9: 47-49.

20. Dreifuss FE. Monitorização intensiva. J Liga Bras Epilep 1990, 3: 58-59.

21. Foster AC, Fagg GE. Taking apart NMDA receptors. Nature 1987, 329: 395-397.

22. Gibson JP, Yarrington JT, Loudy DE, Gerbig CG, Hurst GH, Newberne JW. Chronic toxicity studies with vigabatrin, a GABA-transaminase inhibitor. Toxicol Pathol 1990, 18: 225-238.

23. Gorz AM, Silvado CES, Bittencourt PRM. Barbiturate-refractory epilepsy: safe schedule for therapeutic substitution. Arq Neuropsiquiatr 1986, 44: 225-231.

24. Jawad S, Richens A, Goodwin G, Yuen WC. Controlled trial of lamotrigine (Lamictal) for refratory partial seizures. Epilepsia 1989, 30: 356-363.

25. Kowacs PA, Oliveira TV, Mazer S, Ferreira ZS, Marcourakis T, Bittencourt PRM. Vigabatrin: dose and time-related effects on epilepsy? Epilepsia 1991, 32 (Suppl 1): 12.

26. Loiseau P, Duche B. Carbamazepine: clinical use. In Levy RH, Dreifuss FE, Mattson RH, Meldrun BS, Penry JK. Antiepileptic drugs. New York: Raven Press, 1989, p 533-554.

27. Löscher W, Stephens DN. Chronic treatment with diazepan or the inverse benzodiazepine receptor agonist FG 7142 causes differential changes in the effects of GABA receptor stimulation. Epilepsy Res 1988, 2: 253-259. 
28. Mac Donald RL, Meldrum BS. Principles of antiepileptic drug action. In Levy RH, Dreifuss FE, Mattson RH, Meldrum BS, Penry JK. Antiepileptic drugs. New York: Raven Press, 1989, 59-83.

29. Meldrum BS, Porter RJ. Current problems in epilepsy: new anticonvulsant drugs. London: John Libbey, 1986, p 341.

30. Morselli PL, Bossi L, Munari C. Should EEG recording be included in clinical trials on the new antiepileptic drugs? In Buser PA, Cobb WA, Okuma T. Kyoto Symposia. Amsterdam: Elsevier, 1982, p 482-486.

31. Mumford JP, Dam M. Meta-analisys of European placebo-controlled studies of vigabatrin in drug resistant epilepsy. Br J Clin Pharmacol 1989, 27: 1019-1079.

32. Porter RJ, White G. Objectives and strategy in the search for novel antiepileptic drugs: evaluation in man. In Meldrum BS, Porter RJ. New anticonvulsant drugs. London: John Libbey, 1985, p 46-61.

33. Ragazzo PC, Luccas FJC. Avaliação crítica do EEG no diagnóstico das epilepsias. In Marino R Jr. Epilepsias. São Paulo: Sarvier, 1983, p 31-47.

34. Richens A. Potential antiepileptic drug: vigabatrin. In Levy RH, Dreifuss FE, Mattson RH, Meldrum BS, Penry JK. Antiepileptic drugs. New York: Raven Press, 1989, p 937-946.

35. Riva D. Semiologia das crises epilépticas. In Marino R Jr. Epilepsias. São Paulo: Sarvier, 1983, p 9-16.

36. Rowan AJ, French JA. The role of the eletroencephalogram in the diagnosis and management of epilepsy. In Pedley TA, Meldrum BS. Recent advances in epilepsy. Edinburgh: Churchill Livingstone, 1988, Vol 4, p 63-92.

37. Rowan AJ, Meijer JWA, Beer-Pawlikowski N, Van der Geest P, Meinardi H. Valproate-ethsuximide combination therapy for refractory absence seizures. Arch Neurol 1983, 40: 797-802.

38. Sakamoto AC. Estudo clínico e prognóstico das crises epilépticas que iniciam na infância numa população brasileira. Tese de Doutorado: Faculdade de Medicina de Ribeirão Preto, Universidade de São Paulo. Ribeirão Preto, 1985.

39. Savic I, Widén L, Elander SS. Reversal of benzodiazepine tolerance in epilepsy patients. Epilepsia 1991, 32 (Suppl 1): 10.

40. Schaul N. Pathogenesis and significance of abnormal nonepileptiform rhythms in the EEG. J Clin Neurophysiol 1990, 7: 229-248.

41. Serikawa T, Kogishi K, Yamada J, Ohno Y, Ujihara H, Sasa M, Takaori S. Long-term effects of continual intake of phenobarbital on spontaneously epileptic rat epilepsy. Epilepsia 1991, 319: 9-14.

42. Wilder BJ, Rangel RJ. Phenytoin: clinical use. In Levy RH, Dreifuss FE, Mattson RH, Meldrum BS, Penry

JK. Antiepileptic drugs. New York: Raven Press, 1989, p 233-239. 